\title{
Machine Learning and Multi-Agent Systems in Oil and Gas Industry Applications: A Survey
}

\author{
Khadijah M. Hanga ${ }^{\mathrm{a}, 1, *}$, Yevgeniya Kovalchuk ${ }^{1}$ \\ ${ }^{a}$ Faculty of Computing, Engineering and the Built Environment, Birmingham City University, UK
}

\begin{abstract}
The oil and gas industry (OGI) has always been associated with challenges and complexities. It involves many processes and stakeholders, each generating a huge amount of data. Due to the global and distributed nature of the business, processing and managing this information is an arduous task. Many issues such as orchestrating different data sources, owners and formats; verifying, validating and securing data streams as they move along the complex business process pipeline; and getting insights from data for improving business efficiency, scheduling maintenance and preventing theft and fraud are to be addressed. Artificial intelligence (AI), and machine learning (ML) in particular, have gained huge acceptance in many areas recently, including the OGI, to help humans tackle such complex tasks. Furthermore, multi-agent systems (MAS) as a sub-field of distributed AI meet the requirement of distributed systems and have been utilised successfully in a vast variety of disciplines. Several studies have explored the use of ML and MAS to increase operational efficiency, manage supply chain and solve various production- and maintenance-related tasks in the OGI. However, ML has only been applied to isolated tasks, and while MAS have yielded good performance in simulated environments, they have not gained the expected popularity among oil and gas companies yet. Further research in the fields is necessary to realise the potential of ML and MAS and encourage their wider acceptance in the OGI. In particular, embedding ML into MAS can bring many benefits for the future development of the industry. This paper aims to summarise the efforts to date of applying ML and MAS to OGI tasks, identify possible reasons for their low and slow uptake and suggest ways to ensure a greater adoption of these technologies in the OGI.
\end{abstract}

Keywords:

Machine Learning, Multi-Agent Systems, Artificial Intelligence, Supply Chain Management, Oil and Gas Industry

\footnotetext{
*Corresponding authors

Email addresses: khadijah.hanga@mail.bcu.ac.uk (Khadijah M. Hanga), yevgeniya.kovalchuk@bcu.ac.uk (Yevgeniya Kovalchuk)
} 


\section{Introduction}

Oil is the world's leading fuel accounting for approximately one-third of the total global energy consumption [56]. It is one of the most valuable traded assets in the world, directly or indirectly impacting our daily lives, from necessities such as transportation, heating and electricity, to many other petrochemical products ranging from the things we use to the things we wear [20]. The increase in demand for fossil fuels is on a continuous rise, making it crucial for the oil and gas industry (OGI) to come up with new approaches for improving operations.

The OGI is among the largest, complex and crucial industries in the world, with series of upstream, midstream and downstream activities taking place, including exploration, production, refining, transportation and consumption. The industry is further characterised by working and contractual relationships with governments, joint ventures and other stakeholders. It is highly dynamic in nature, making it difficult for the traditional centralised approach to work, especially with the rise in global demand [20]. Attempts to identify innovative approaches to process and manage information for the purposes of enhancing operational potency, reducing operating costs and boosting profits necessitate advancements in technologies within the industry. For example, real-time data streams continuously generated by sensors are utilised to ensure better control and optimisation of crude production [25]. Robotics is used in offshore fields for drilling, inspection and damage control to enhance efficiency and personal safety [49]. Wireless sensor networks are used to monitor and enhance production, as well as detect and prevent issues with regards to health and safety [46]. The radio-frequency identification (RFID) technology is used for asset management, oil rig site management, pipeline inspection, safety and security [15].

As the number of technologies for data processing and management increases, their control requires decentralisation with such features as autonomy, intelligence and logic to ensure their smooth and coordinated functioning across multiple tasks, including scheduling, manufacturing, control, diagnosis and logistics. To achieve such intelligent and decentralised control, many researchers are inclined towards machine learning (ML) and multi-agent systems (MAS). A typical MAS is made of several interacting agents, where an agent is a computer system proficient in autonomous activities to achieve certain tasks in an environment [58]. The ability of agents to interact with each other through negotiation, collaboration and coordination, while adapting to changes and disruptions, makes them suited for distributed control. Due to its conformity with the complex and distributed nature of the OGI, the MAS approach has gained attention of researchers as a means to solve a number of tasks in the OGI. To facilitate the development of resilient agents, especially in unpredictable, diversified environments, ML algorithms are essential when it comes to providing reputable strategies. While there are not many examples of combining ML with MAS in OGI applications to date, this is what may be required in the future for the industry to maintain its efficiency at the time of rapid technological development and a wider adoption of alternative energy.

This paper provides an overview of the existing ML and MAS solutions for the OGI, identifies the challenges preventing their real-life deployment and summarises their benefits to encourage a wider adoption. The survey is arranged as follows. Section 2 presents an 
overview of the OGI, the tasks involved and technology adopted in the industry. Sections 3 and 4 introduce ML and MAS and review their applications to the OGI tasks, respectively. Section 5 compares ML and MAS approaches to OGI tasks and discusses possible ways and advantages of combining the two approaches for their greater adoption in the OGI. Section 6 highlights challenges and future directions in this respect, while Section 7 concludes the survey.

\section{The Oil and Gas Industry}

\subsection{Overview}

The OGI is an extremely large industry involving upstream, midstream and downstream sectors. It is a complex but essential industry worldwide. It directly or indirectly impacts our daily lives enabling services such as transportation, heating and electricity, as well as many petrochemical products from things we use to those we wear [20]. No wonder that petroleum is often called "black gold" by virtue of its worth and value in human life and global economy [27].

Presently, OGI organisations are considering new ways of cutting operations cost, enhancing efficiency and increasing out-turn. Challenges and risks associated with activities and operations throughout the supply chain require to be addressed profoundly, which compels the advancement of technology within the OGI, especially for data processing and management. Some examples of such technologies include digitisation of oil fields, real-time optimisation of drilling operations, the use of nanotechnology, wireless sensor networks to aid gauging, reservoir modelling and diagnostics [16]. Research in the industry predicts ample resources left to sustain current oil production levels for at least 50 more years [56]. Thus, the main challenges facing the OGI are ensuring an increase in productivity and conveying the products to end users at the least possible cost [10]. Considering its global nature and complications of engaging in contract relationships with governments, joint ventures and other stakeholders, the OGI also requires technical resolutions for a more effective data management.

\subsection{Tasks}

Supply chain in the OGI is subdivided into three closely related phases, each involving a series of operations. These phases include the upstream, midstream and downstream. The upstream mainly involves exploration and production of crude. The midstream extends both upstream and downstream and involves storage and transportation; however, it is often ignored when listing out the OGI supply chain phases. The downstream is responsible for oil and gas refining, as well as distributing the end products. Each level of the supply chain is associated with complexities that require strategic, tactical and operational decision making [54].

Following the exploration, production is the first step in the OGI supply chain; it involves drilling, extraction and recovery of oil from beneath the earth. During production, crude oil is produced both on land (onshore) and in shallow waters (offshore). To accomplish successful production of crude oil and natural gas, different equipment is utilised and 
various production operators are involved being responsible for organising and supervising all operations in searching for and extracting oil [10].

Crude oil production platforms, whether offshore or onshore, are full of huge risks. As such, effective control and maintenance are required to ensure efficient and reliable production processes, higher productivity, longer equipment life, safety of people and the environment, and staying within budget. Control involves applying planning and sustenance abilities to ensure improved performance and reduce the risk of failure at every stage of the oil and gas supply chain. Maintenance includes regular inspection and examination of operating conditions for taking preventive, predictive and corrective measures, as well as making strategic, tactical and operational decisions [38].

Supply chain management (SCM) ensures making plans, coordinating activities and exploring improvements. In the OGI, SCM faces several challenges, mostly in terms of logistics and attitude in respect to teamwork and information sharing within the supply chain partners. The improved supply chain in the OGI requires new principles for collaboration [19].

Another important but underdeveloped area of study among the multiple accomplishments in the OGI is the monitoring, processing and management of information. This encompasses data collected from the fields, amount of oil and gas produced and exported. Traditional methods such as centralised databases often fail since storing, analysing and sharing collected data require decentralisation and continuous verification for consistences across various stages of the supply chain. Some traditional methods may be adequate for reporting the state of the environment around the field or the quantity of produced and distributed oil; however, they cannot provide the required real-time and future support such as preventing data being wrongly reported or tampered at different stages of its processing.

\subsection{Technology}

The use of computer-aided techniques to assist businesses is becoming standard in industries, including the OGI. To maintain a sustainable and profitable business, the OGI has to explore new technologies that can drive its efficiency. It is thus crucial to find out what advantages a technology can offer over others for a certain task [51]. The demand for safety in the OGI, along with a keen interest in intelligent fields, smart wells and real-time analysis, brought about a hype in the exploration of different kinds of technology that are powerful, intelligent and robust in nature [33].

Autonomy becomes very significant in environments, where risks are high or humans are not capable of certain actions, or when trying to be cost-effective. With the increase of the industry size, things like collecting data, assessing it and making decisions in fractions of seconds are impossible to human operators. Setting overall goals and delegating decisionmaking to autonomous systems is the best humans can do in such situations.

Among many recent technologies, the Internet of things (IoT) has a big impact on the OGI development. For example, [43] outlined some of the IoT applications in the OGI, which included the use of wearable watches, smart helmets and smart glasses by oil field engineers in offshore fields for real-time assistance, safety and communication with the control room for navigation and enhanced collaboration. Drones are used for inspection of leakages in pipelines and flare emissions, while smart pipelines are employed for detecting damages and 
sending real-time data to the control room. Robots are used in offshore fields for drilling, inspection and damage control to enhance efficiency and personal safety [49]. Micro- and nanotechnologies have contributed significantly to several areas of the OGI to give detailed and more accurate information [25]. The OGI is considered as one of the pioneering sensorbased industries. For example, wireless sensor networks are used to wirelessly control and oversee oil assets, including pipeline leakage, rust, equipment and reservoir status in real-time [46]. The radio frequency identification (RFID) technology is used for asset management, oil rig site management, pipeline inspection, safety and security [15]. The data generated from various sensors can potentially provide insights to assist the OGI to improve operations ans safety, prevent problems and reduce operational costs. This data is usually massive and difficult to make sense of, especially by human operators. Intelligent systems are the only currently available solution capable of bringing real-time analysis and decision-making [33].

\section{Machine Learning in Oil and Gas Industry Applications}

Just as humans learn by doing tasks repeatedly and making mistakes, machines too are able to learn and improve their performance with the aid of ML [41]. The main goals of ML are making predictions, performing clustering, extracting association rules and making decisions from a given information or data [34]. ML has a great potential in OGI applications, especially when it comes to analysing and interpreting data. It offers better ways of developing drilling plans, diagnosing, monitoring, predicting and performing real-time optimisation at a minimal cost. ML algorithms that have been effectively applied to OGI tasks include Support Vector Machine (SVM), Artificial Neural Networks (ANN), Deep Learning (DL) and Genetic Algorithms (GA). Applications using these algorithms are reviewed below in turn.

\subsection{Production}

ML can help to improve the oil and gas production rate and minimise the cost of lifting. It has been effectively used for enhancing reservoir modelling and preventive maintenance. In particular, ML models have been proposed for determining optimal times for outlined maintenance so as to avoid failures and optimise the regular maintenance operation schedule. Below, we review the studies applying ML to various problems associated with oil and gas production.

Qiao et al. [44] introduced an oil and gas production forecasting model, which combined the Particle Swarm Optimisation (PSO) and Least Squares Support Vector Machine (LSSVM) methods. Using historical data of an oil and gas company and simulations run in MATLAB, the authors were able to analyse the factors impacting the production and build an accurate prediction model that was trained at a high speed.

Panja et al. [40] developed and compared an LS-SVM model, an Artificial Neural Networks (ANN) model and a Response Surface model (RSM) to predict the oil production and gas-to-oil ratio considering reservoirs that are subjected to time and rate related con-

straints. Some fundamental factors were considered as input parameters. The same datasets 
were used to train the three models for predicting the production under different scenarios. According to the test results, the LS-SVM model demonstrated the highest accuracy with respect to the gas-to-oil ratio prediction. It was concluded by the authors that the application of LS-SVM to various production and reservoir engineering tasks is promising.

Chaki et al. [9] applied the Modular ANN (MANN) approach to determine the fraction of sand in a reservoir. Three seismic properties, namely, the seismic impedance, instantaneous amplitude and frequency, were used. The reservoir and seismic data for this study were gathered from a hydrocarbon field in the western onshore of India. Three networks were designed for three separate zones. The sand fraction and three seismic attributes from seven wells were used to train and test the ANNs. The sequence correlating to the remaining well was used for blind prediction. The proposed framework gave good predictions with a short program completion time and enhanced visualisation through post-processing of the forecast sand fraction.

Most recently, DL has demonstrated the potential to reform the stakes of oil and gas companies facing profit-making pressure [8]. DL algorithms can reveal patterns and information not easily seen by humans; they can automatically detect risks in oil pipelines, thus minimising infrastructure risks. They can spot anomalies, which traditional rule-based electronic condition monitoring systems miss, and alert rig operation command centres in a timely manner. To improve subsurface characterisation, two DL models were developed to predict the nuclear magnetic resonance for $\mathrm{T} 2$ time distribution from conventional well logs [29]. The authors implemented a Variational Auto-Encoder (VAE) in combination with a Convolutional Neural Network (CNN) to effectively extract features from the T2 distribution, and a Long Short-Term Memory (LSTM) network to extract short- and long-term dependencies from the T2 distribution. The data were collected from the Bakken Shale Formation, and the models were trained using the same training and testing datasets. Results demonstrated that the prediction accuracy for the two models was good overall and both models were robust to noise.

Finally, GA has been successfully applied to the development of oil fields, scheduling production, characterisation of reservoirs and seismic inversion [55].

\subsection{Anomaly detection}

Another significant application of ML to the OGI is anomaly detection; ML can help to identify patterns and predict failures based on relationships between valid readings.

Jin et al. [22] used LS-SVM to detect leak levels on a gas pipeline based on the acoustic wave method. The authors experimented on a test bed comprising a gas pipeline, some compressors, valves for regulation, acoustic data retrieval and leakage simulation systems to simulate different leakage levels. Acoustic signals were gathered under each of the normal, small, medium and large leakage conditions. Results from the experiment demonstrated that the LS-SVM model can identify distinct leakage levels based on the collected signals within a short time and with a high accuracy. 


\subsection{Price prediction}

In countries where economy strongly depends on oil production and export, predicting oil prices is impractical using traditional forecasting models. Kristjanpoller and Minutolo [26] proposed the ANN-Generalised Autoregressive Conditional Heteroscedasticity (ANNGARCH) method to predict the volatility of fuel prices. In this method, the ANN model takes an input from estimates of the GARCH forecast and financial time-series data, and uses it to determine the instability of oil prices. The authors made use of the exchange rate and stock market index differences to enhance the prediction of crude oil prices. Three different forecasting scopes were applied to analyse and amplify the forecasting capacity of the model. In addition, a loss function evaluation criterion was used to determine the progress of the volatility prediction. The datasets analysed in this study came from the Oil Spot Price and Oil Futures Price by Bloomberg for the period from July 2002 to May 2014. The results indicated that the proposed method improved the GARCH model predictions of the oil spot and futures price volatility using the 21-days window.

\section{Multi-Agent Systems in Oil and Gas Industry Applications}

Multi-agent systems (MAS) have been defined using several terms by various authors due to their extended features. Going by one of the most common definitions, MAS is made up of various interacting agents, where an agent is a computer system with the ability to perform autonomous actions in order to accomplish certain goals in some environments [58]. An intelligent agent achieves its goals by perceiving its environment and utilising observations to decide on which action(s) to perform.

MAS offers a chance to amplify production systems that are decentralised, emergent and concurrent. It is an inspiring and promising way of managing and using distributed computing and information systems [57]. MAS are especially suited for organisations with different or conflicting goals, which can be broken down into several independent tasks and assigned to agents. They can also benefit systems whose capacity and specifications are likely to change over time. MAS are successfully applied to a vast range of disciplines such as electronic commerce and information management in graphics and transportation [6]; logistics, controlling processes (e.g air traffic control), manufacturing, tele-communication, smart grids and robotics [31]; electric power systems [30]; patient monitoring and rescue team management [57], [28]. MAS have also been utilised in the OGI to improve operational efficiency and solve impending issues. Some of such applications are reviewed below, grouped into three categories based on the core OGI tasks reviewed in Section 2.2: production, maintenance and SCM.

\subsection{Production}

To maximise the amount of oil produced and limit the volume of water and sand used in oil production, Engmo and Hallen [13] proposed a MAS for the control and optimisation of oil production. The authors made use of the Prometheus design tool [53] to build their agent system with several agents overseeing the production based on some tasks allocated to them. Particularly, the agents monitored and analysed sensor values for the wells and the 
processing plant. An agent was assigned to represent the operator of the control room, and another to represent all agents in the field. The authors developed an application to test their agent system, where they simulated a simple oil field comprising of a few wells and a processing plant. The JACK intelligent agents environment [7] was used in this study to build, run and integrate the MAS, while the Eclipse software development kit was employed for the system implementation. For the test run, the authors generated values of three relatively random variables affecting the production, which included the permeability, water and sand ratios. They operated the simulator with a control system to compare a human operator and the agent system. They also did the same without the control system to enable

standard comparison. While both the human operator and agent system reduced the amount of time used in critical circumstances and increased the quantity of oil produced, the agent system achieved a higher production level compared to that of the human operator system. The authors concluded that their agent system can be relied upon to manage some tasks in the industry and can perform better than human operators.

To resolve conflicting control concerns and comply with the dynamic nature of oil fields, Mikkelsen et al. [32] developed an extensible multi-objective control model for offshore oil and gas production. They implemented a stratified MAS, in which control objectives were assigned and managed by various agents through a uniform negotiation context at three layers, namely, the strategic, tactical and operational decision layers. Each agent accessed historical production data from the DONG Energy EP-operated Siri oil and gas field in the North Sea and online data feeds to achieve a goal. A negotiation process was used to coordinate the agents' actions, while a self-reflecting implementation approach was employed to prevent agents from becoming dominant. Two experiments were conducted, from which the authors observed that their approach improved the system performance compared to a manually operated system. They concluded that their proposed system can meet the dynamic operating conditions of oil and gas fields to conform to the present and future requirements with a reduced environmental impact and greater economic pay-offs.

Dobrescu et al. [12] presented a MAS framework for shared control of petroleum services for onshore oilfields. The authors proposed a distributed control strategy for a basic production system based on the IEC 61499 standard. They used an existing design architecture for distributed control and assigned requirements for the MAS to carry out control tasks as a service. The traditional production units were transformed to production unit agents to ensure system dynamics and a better load distribution. The authors adapted the remote execution process for the MAS so as to identify a possible need for modification at the supervisory level. In the future, they plan to implement the approach for a real situation and evaluate its performance in the presence of operational unpredictability.

\subsection{Safety and Maintenance}

A growing demand for proactive measures to protect oil pipelines from vandals and leakages, which are popular mostly during crude oil conveyance, has attracted attention of several researchers. Ahmed et al. [1] proposed a multi-agent-based approach for preventing and controlling oil pipeline vandalism. The authors used MAS engineering to design a system comprising reactive and proactive agents working together to monitor and avert dangers in 
a pipeline. According to this MAS architecture, the Intelligent Agent detects abnormal pressure and alerts another agent, called the Mobile Agent, with the details and location of the found abnormality. At the same time, the Sensor Agent detects metallic objects in the pipeline and alerts the Master Agent, which represents the control centre, through the Mobile Agent. The Protective Agent provides the first-hand protection to the pipeline whenever the presence of any metallic object is discovered before the arrival of security guards. The proposed approach has not been implemented, but the authors believe that the collaboration expressed by the agents is a good sign that the proposed framework is suited for supporting the case of pipeline vandalism.

Ramirez et al. [45] proposed an intelligent supervisory MAS-based system with multiple feedback controls for industrial processes. The authors combined some fuzzy logic rules, an internal model control architecture and a MAS to accomplish two petroleum industrial operations: gas-oil separation and oil heating. Standard local interconnected supervising schemes were joined to form an overall supervisor so as to meet local goals in each of the processes. The design of the local supervising agent consisted of two levels: the regulatory level with an internal model control architecture and supervisory level. The latter includes an event detector developed using fuzzy logic [36] and a decision system that uses information received from the event detector to make appropriate decisions. The global supervising agent was designed based on a predefined mechanism to guarantee the accomplishment of the global objective of the entire process. Computational simulations for the emulation of the local and global supervising agents were carried out, and the results demonstrated a possibility of reaching the entire process global objective owing to the cooperation between local supervising agents. The authors highlight that the security of all processes is more efficient when applying the proposed distributed supervision compared to that of a centralised system. For future development, the authors suggest to include another process for identifying an agent able to manage conflicts to improve the collaboration between the local supervising agents.

Oliveira et al. [11] proposed the SGCP (a Portuguese acronym referring to a MAS for oil field management). The SGCP was used to simulate an oil field, situated in Bahia, Brazil. The oil field components were monitored by agents through interaction with a database containing real data from the field. The gathered data included sensor readings for every field and proficient knowledge about costs. The agents applied the information to estimate the present state of every single oil field component and the entire field. They all acted according to their assigned functions, e.g. operation, supply and intervention. The agents also interacted with each other to achieve an improved global profit. Problems were identified through a statistical calculus approach, which included analysing the level of oil production. The authors integrated the SSCP into a tool that had prior understanding of the oil field conditions and aided decision making in an intelligent way to make inferences based on rules and fuzzy logic. The agents were implemented using the Java Agent Development Environment (JADE) framework [5], while SGCP ontology was developed using the Protege 4.2 software and the Java class Ontology.java supported by the JADE framework. The methodology for storing and integrating expert knowledge (MAICE) [37] was used to define the action rules for the agents. During each simulation, environment information was stored 
in a MySQL 5.0 database connected to SGCP. Information was channelled from one agent to another using FIPA's agent communication language. The outcome indicated that using MAS in the oil field operation encouraged the agents to act together in analysing problems, thus cutting down the unnecessary expense of digging into raw data in various systems in search of solutions.

A MAS for network management (NM-MAS) comprising agents with different capacities for resolving network fail-over was presented by Ioniţă and Ioniţă [21]. For the experiment, a gas-oil separation plant (GOSP) was mapped to some implemented industrial wireless network components. The GOSP operator made use of high-level attainment information of network elements to identify faults that may occur. NM-MAS and GOSP communicate in phases with the help of agents, whose assigned activities include; symptom detection, failure recognition, diagnosis and repair planning. Two evaluation metrics, namely, the success rate of plan repair and fault-tolerance network indicator, are used to measure the NM-MAS performance. The authors plan to implement the agents as part of a future work. They also highlight the drawbacks of using wireless network, the outcome of which can affect the accuracy of data, thus making real-time control difficult or impossible.

Based on an established research towards a feasibility study of an intelligent asset management system for the offshore OGI in Atlantic Canada, Sayda and Taylor [47] proposed a MAS called ICAM to control and manage assets in the petroleum industry. Having previously defined a conceptual model, architecture and implementation plan for the proposed system in an existing work, the authors came up with a general structure of the ICAM system with an intelligent supervising agent and discussed the knowledge representation and processing requirements. They defined the agent structure, communication protocols and AI requirements for the component agents. The authors conducted a real-time simulation experiment on the ICAM system model to assess and validate its performance, which indicated positive improvements in the design and development of the MAS. These authors further presented three different papers fully addressing the ICAM system design and development [48]. To analyse the behaviour of reactive agents, plant data was gathered and examined for every reactive agent during simulations. The outcome from the simulation showed that the ICAM system prototype behaviour conferred to its design conditions. To analyse the ICAM performance, the authors conducted a real-time simulation experiment based on the system's logical behaviour and its reaction to the dynamics of the outside environment. An impressive logical behaviour was observed; the system reacted to simple faulty sensors and actuators. The overall ICAM performance analysis outcome was good. Finally, the authors further tested the system in two different simulation scenarios to investigate the MAS behaviour during unforeseen plant disruptions and conditions, which resulted in discovering some limitations in the ICAM system prototype design. Suggestions on how to overcome these limitations and enhance the ICAM system performance were outlined in the paper.

\subsection{Supply Chain Management}

The increase in global demand along with the inflexible and dynamic nature of the OGI has made its SCM more complex and challenging compared to that of many other industries [19]. Unfortunately, research work on the MAS approach to the complete supply chain in 
the OGI is scarce. The majority of the existing studies focus only on one of the supply chain aspects such as logistics planning or process optimisation.

Sinha et al. [50] proposed a combination of MAS and co-evolutionary PSO based on the Cauchy distribution to manage a petroleum supply chain. In the system, supply agents are guided by supporting agents who coordinate themselves to optimise the cost of a specified subsystem, thereby reducing the entire cost of the supply chain. A mathematical formula was used to optimise the resource allocation to each agent. The simulation outcome showed that applying the MAS favoured the maintenance of optimal crude oil quantities in the inventories of the considered refineries. The authors observed that the Cauchy distribution approach had a fair impact on the MAS resource requirement optimisation.

Julka et al. [23] proposed a petroleum refinery integrated supply chain modeller and simulator (PRISMS) to assist in tendering decision support during crude oil procurement operations. The authors used a unified agent-based modelling framework in an object oriented way to build the PRISMS for the entire supply chain network. According to this framework, information and resources are modelled as objects, supply chain entities - as agents, and the internal departments of the entities - as sub-agents. Information flow is represented by the transfer of objects among the agents. Based on the framework, the proposed decision support system can work in two different modes, namely, the simulation and analysis modes, with one complementing the other. Using the agent-based approach, the PRISMS simulates certain refinery departments such as procurement, logistic, storage and sale operations. During a set up, a certain operation in the refinery is simulated for a given number of days. The entire data, which includes messages, events and results, are stored as features of a particular agent during the simulation. To illustrate the effectiveness and practicality of the PRISMS for SCM, the authors considered policy changes, external changes and modification of plant configuration as case studies. In the future, the authors plan to embed detailed solution approaches used by respective departments and study their capability in the entire refinery; experiment on more case studies such as those covering oil sales and long-term contract checks; extend the approach, by making it capable of monitoring and handling exceptions in the supply chain.

\section{Analysis and Discussion}

\subsection{Comparison of Machine Learning and Multi-Agent System Approaches to Oil and Gas Industry Tasks}

Both ML and MAS approaches can be applied interchangeably to some OGI tasks. For example, while LS-SVM was used to detect leak levels on a gas pipeline based on the acoustic wave method [22], a MAS-based approach was used to monitor and avert dangers, which included leakages in pipelines [1]. Another example is where GA [55] and MAS [53] were applied to control and optimise the oil production. At a closer look, it can be gathered that while both ML and MAS approaches facilitate decision-making, they do this in different ways and at different scales. In particular, ML is often applied to make predictions such as predicting production volumes and oil prices, detecting anomalies, as well as optimising and scheduling production. On the other hand, MAS are typically used to automate and 
coordinate complex and distributed tasks across the entire supply chain. They make good supervisory systems, while the agent communication and collaboration is well-suited for oil-field, network and asset management. From the acceptance point of view of the two approaches, it is clear that ML is a more extensively studied area and is gaining acceptance in the OGI due to its popularity in many other fields. On the other hand, as promising as MAS is, it still has not attained the deserved attention in the OGI; it is yet to be fully explored and experimented in reality.

\subsection{Combining Machine Learning and Multi-Agent System Approaches}

Considering the potential of both ML and MAS, level of maturity and acceptability of ML and suitability of MAS for solving complex, unpredictable problems, merging the two approaches can produce a desirable solution for OGI applications. In particular, when modelling agent systems, it is impossible to forecast the entire circumstances an agent may experience, especially in dynamic environments. It is thus impractical to define an agent's ideal behaviour beforehand. Therefore, in a multi-agent setting, agents would benefit from having the ability to learn from and adjust to their environment [4]. Furthermore, complexities can arise in the interaction between agents as their number and behavioural sophistication increase. ML can assist agents to automatically adjust their co-behaviour as the complexity of a MAS environment becomes challenging [39]. Out of the three main ML approaches, namely, supervised, unsupervised and reinforcement learning, the latter is the most utilised in agent learning because it matches the agent paradigm explicitly [24]. However, only a few OGI applications employ reinforcement learning. Instead, the majority of the existing MAS-based applications in the OGI use supervised or unsupervised ML algorithms for predictive or descriptive analytics to inform artificial and human agents about a certain aspect of the complex system (e.g. predict production or pipe leakage). In other words, ML models are embedded in MAS as stand-alone entities that parse incoming data rather than facilitate agent learning.

As an example of a MAS based on reinforcement learning, Aissani et al. [2] proposed a multi-agent model for dynamic planning of maintenance tasks for a petroleum industry production set up. As a case study, the authors used the Arzew RA1Z refinery in Algeria. Considering the dynamic nature of the petroleum refinery, the uncertainty and unforeseen nature of the environment, the modular Markov decision process (MDP) architecture was employed in the system. The authors modelled multiple agents organised in a hierarchy capable of learning from experience using the state-action-reward-state-action (SARSA) algorithm [52] to ensure efficient maintenance planning and continued development of the solution standard. The SARSA algorithm allows evaluation during learning by considering changes in the feedstream as a performance indicator. The Contract Net Protocol was used for the agent interaction. The system was developed using the multi-agent development kit (MaDKit) platform [18] and implemented in Borland JBuilder. Experimental results proved that the method can provide online scheduling solutions for predictive and corrective maintenance tasks and improve their nature by minimising out flow alterations from the tanks. At the same time, scheduling maintenance tasks was not always achieved for the off-peak pump times, especially in the case of unexpected failures, because the authors 
did not consider some precautionary maintenance tasks that ought to be implemented on time. As a future work, the authors proposed widening the time horizon of experimentation while considering more complex maintenance tasks and applying the prototype to other oil production units. They also highlighted that using distributed systems with a powerful calculation approach could be beneficial.

As an example of incorporating supervised ML models into MAS, Eze et al. [14] proposed an integrated oil and gas pipeline monitoring and incident mitigation system (IOPMIMS), which uses the distributed systems and case-based reasoning approach to offer proactive protection to pipelines. The authors proposed a wireless sensor network node architecture that has the capacity to gather signals from all sensors. A MAS embedded into Antronix Fiber Nodes (AFN) fuses the data based on the signals at the allotted sub-station and base station to confirm if certain events pose threat to a pipeline. At the base station, the MAS collates data from different wireless devices, which facilitates decision making by the system. Upon a risk or leak detection in the pipeline, alerts are being forwarded to the security personnel or pipeline operators. Data from the Concawe group's website was collected and simulated to investigate the performance of the proposed system. Three machine learning algorithms (ANN, SVM and Decision Tree) were used for data fusion and decision support. Out of the three algorithms, SVM achieved the best performance. While demonstrating the potential of the proposed system, the paper highlighted some challenges that should be addressed in the future such as low battery life and low bandwidth.

\section{Current Challenges and Future Directions}

While the research community regains interest in MAS due to the recent advancements in AI and deep reinforcement learning [17], there are not many examples of MAS-based applications deployed in industries including the OGI. Müller and Fischer [35] attribute the poor uptake of MAS beyond simulation environments to lack of funding as funding bodies are focused on other approaches such as service-oriented, grid, autonomic and cloud computing. Leitao [28] suggest that the major limiting factors are inevitable early investments, the need to embrace a distributed reasoning, missing standardisation, various real-time constraints and lack of maturity in the technology. Alhosani and Zabri [3] mention that the OGI lacks the structure to bring together, promote, reuse and manage knowledge due to the scattered nature of information. Other reasons for the weak industrial adoption of MAS as outlined by Pěchouček and Mařík [42] include the following:

- lack of awareness of the accomplished MAS in industries, wrong impressions about their technical capacity and high expectations by pioneer researchers, which often lead to frustration and loss of interest;

- lack of adequate knowledge on the potential of the agent technology in the industry;

- shortage of development tools fit for efficient industrial implementation;

- risks associated with the acceptance of new technologies not confirmed as working or beneficial in big industries. 
On the other hand, problems such as depletion of oil and gas reserves, price increase, theft and vandalism, lack of transparency and accountability, force the OGI decision makers to look into the advantages that AI can offer in handling the ever increasing amount of data for prediction, prevention and optimisation. To spread the investments and minimise the risks, a staged approach can be adopted. In particular, separate predictive and descriptive ML models for specific tasks developed at different times can be assigned to individual agents, eventually resulting in a scalable MAS overseeing the entire OGI operation through agent co-operation.

\section{Summary}

This paper reviewed the existing studies employing machine learning (ML) and multiagent systems (MAS) to solve various tasks in the oil and gas industry (OGI). The OGI is very complex consisting of upstream, midstream and downstream sectors and involves many stakeholders ranging from government and regulatory bodies, to joint ventures and consumers. In addition, the industry generates and relies on a huge amount of data. ML can be an important tool for mining patterns and information from the data and generating predictions to assist decision-making, while MAS have the potential to help manage the dynamic, distributed and uncertain relationships in the industry. Several studies have proposed ML- and MAS-based solutions for the OGI. However, the majority of them are focused on a specific task such as production, control or maintenance rather than automating the entire supply chain and information governance. Furthermore, virtually all of the proposed MAS-based solutions are limited to simulated environments only, without being deployed or used in real life. Things like high investments and risks associated with implementation and deployment, as well as lack of standards and appropriate developmental tools, prevent the uptake of the technologies in the industry. As a way forward, the OGI leaders and management can consider adopting a staged approach for embedding ML and MAS into the existing business processes to spread the costs and risks. In particular, separate ML predictive and descriptive models developed over time for various OGI data processing and management tasks can eventually be combined within a MAS that would orchestrate the different models. After that, the intelligent ability of the agents constituting the MAS and their co-operation can be improved by incorporating state-of-the-art deep reinforcement learning techniques. Finally, opening up data and sharing practices can speed-up the development and adoption of ML- and MAS-based solutions for the OGI, which ultimately would bring benefits for everyone involved in the industry.

\section{References}

[1] Ahmed, M., Umar, A., Bute, M., 2017. Multi-agent based architectural framework for the prevention and control of oil pipeline vandalism. In: Computing Networking and Informatics (ICCNI), 2017 International Conference on. IEEE, pp. 1-8.

[2] Aissani, N., Beldjilali, B., Trentesaux, D., 2009. Dynamic scheduling of maintenance tasks in the petroleum industry: A reinforcement approach. Engineering Applications of Artificial Intelligence $22(7), 1089-1103$. 
[3] Alhosani, A., Zabri, S. M., 2018. A uniform supply chain management framework for oil and gas sector: A preliminary review. International Journal of Advanced and Applied Sciences 5 (2), 19-24.

[4] Alonso, E., D'inverno, M., Kudenko, D., Luck, M., Noble, J., 2001. Learning in multi-agent systems. The Knowledge Engineering Review 16 (3), 277-284.

[5] Bellifemine, F., Caire, G., Greenwood, D., 2007. Developing Multi-Agent Systems with JADE. John Wiley \& Sons.

[6] Burmeister, B., Haddadi, A., Matylis, G., 1997. Application of multi-agent systems in traffic and transportation. IEE Proceedings-Software 144 (1), 51-60.

[7] Busetta, P., Hodgson, A., Lucas, A., 1999. Jack intelligent agents - components for intelligent agents in java. AgentLink News.

[8] Business, A., 2017. Deep learning taking oil and gas industry by storm. URL https://aibusiness .com/deep-learning-taking-oil-gas-industry-storm/

[9] Chaki, S., Verma, A. K., Routray, A., Mohanty, W. K., Jenamani, M., 2014. Well tops guided prediction of reservoir properties using modular neural network concept: a case study from western onshore, india. Journal of Petroleum Science and Engineering 123, 155-163.

[10] Chima, C. M., Hills, D., 2007. Supply-chain management issues in the oil and gas industry. Journal of Business \& Economics Research 5 (6), 27-36.

[11] de Oliveira, V. L. C., Tanajura, A. P. M., Lepikson, H. A., 2013. A multi-agent system for oil field management. IFAC Proceedings Volumes 46 (7), 35-40.

[12] Dobrescu, S., Chenaru, O., Matei, N., Ichim, L., Popescu, D., 2016. A service oriented system of reusable algorithms for distributed control of petroleum facilities in onshore oilfields. In: Electronics, Computers and Artificial Intelligence (ECAI), 2016 8th International Conference on. IEEE, pp. 1-6.

[13] Engmo, L., Hallen, L., 2007. Software agents applied in oil production. Master's thesis, Institutt for datateknikk og informasjonsvitenskap.

[14] Eze, J., Nwagboso, C., Georgakis, P., 2017. Framework for integrated oil pipeline monitoring and incident mitigation systems. Robotics and Computer-Integrated Manufacturing 47, 44-52.

[15] Felemban, E., Sheikh, A. A., 2013. Rfid for oil and gas industry: Applications and challenges. International Journal of Engineering and Innovative Technology (IJEIT) 3 (5), 80-85.

[16] Fjellheim, R., Landre, E., Nilssen, R., Steine, T. O., Transeth, A. A., 2012. Autonomous systems: Opportunities and challenges for the oil and gas industry. Norwegian Society of Automatic Control.

[17] Foerster, J. N., de Freitas, N., Assael, Y. M., Whiteson, S., 2016. Learning to communicate with deep multi-agent reinforcement learning.

[18] Gutknecht, O., Ferber, J., 2000. The madkit agent platform architecture. pp. 48-55.

[19] Hussain, R., Assavapokee, T., Khumawala, B., 2006. Supply chain management in the petroleum industry: challenges and opportunities. International Journal of Global Logistics and Supply Chain Management 1 (2), 90-97.

[20] Inkpen, A. C., Moffett, M. H., 2011. The global oil and gas industry: management, strategy and finance. PennWell Books.

[21] Ioniţă, L., Ioniţă, I., 2014. Nm-mas: A multi-agent system for network management in oil industry. In: RoEduNet Conference 13th Edition: Networking in Education and Research Joint Event RENAM 8th Conference, 2014. IEEE, pp. 1-6.

[22] Jin, H., Zhang, L., Liang, W., Ding, Q., 2014. Integrated leakage detection and localization model for gas pipelines based on the acoustic wave method. Journal of Loss Prevention in the Process Industries $27,74-88$.

[23] Julka, N., Karimi, I., Srinivasan, R., 2002. Agent-based supply chain management2: a refinery application. Computers and chemical engineering 26 (12), 1771-1781.

[24] Khalil, K. M., Abdel-Aziz, M., Nazmy, T. T., Salem, A.-B. M., 2015. Machine learning algorithms for multi-agent systems. In: Proceedings of the International Conference on Intelligent Information Processing, Security and Advanced Communication. ACM, p. 59.

[25] Kong, X., Ohadi, M., et al., 2010. Applications of micro and nano technologies in the oil and gas industry-overview of the recent progress. In: Abu Dhabi international petroleum exhibition and con- 
ference. Society of Petroleum Engineers.

[26] Kristjanpoller, W., Minutolo, M. C., 2016. Forecasting volatility of oil price using an artificial neural network-garch model. Expert Systems with Applications 65, 233-241.

[27] Lalude, G., 2015. Importance of oil to the global community. Global Journal of Human-Social Science Research 15 (2).

[28] Leitao, P., 2013. Multi-agent systems in industry: Current trends and future challenges. In: Beyond Artificial Intelligence. Springer, pp. 197-201.

[29] Li, H., Misra, S., 2018. Long short-term memory and variational autoencoder with convolutional neural networks for generating nmr t2 distributions. IEEE Geoscience and Remote Sensing Letters 16 (2), 192 195.

[30] McArthur, S. D., Davidson, E. M., Catterson, V. M., Dimeas, A. L., Hatziargyriou, N. D., Ponci, F., Funabashi, T., 2007. Multi-agent systems for power engineering applicationspart i: Concepts, approaches, and technical challenges. IEEE Transactions on Power systems 22 (4), 1743-1752.

[31] Merabet, G. H., Essaaidi, M., Talei, H., Abid, M. R., Khalil, N., Madkour, M., Benhaddou, D., 2014. Applications of multi-agent systems in smart grids: A survey. In: Multimedia Computing and Systems (ICMCS), 2014 International Conference on. IEEE, pp. 1088-1094.

[32] Mikkelsen, L. L., Jorgensen, B. N., et al., 2012. Towards intelligent optimization of offshore oil and gas production using multi-agent software systems. In: SPE Western Regional Meeting. Society of Petroleum Engineers.

[33] Mohaghegh, S. D., et al., 2005. Recent developments in application of artificial intelligence in petroleum engineering. Journal of Petroleum Technology 57 (04), 86-91.

[34] Mohammed, M., Khan, M. B., Bashier, E. B. M., 2016. Machine learning: algorithms and applications. Crc Press.

[35] Müller, J. P., Fischer, K., 2014. Application impact of multi-agent systems and technologies: A survey. In: Agent-oriented software engineering. Springer, pp. 27-53.

[36] Neuroth, M., MacConnell, P., Stronach, F., Vamplew, P., 2000. Improved modelling and control of oil and gas transport facility operations using artificial intelligence. In: Applications and Innovations in Intelligent Systems VII. Springer, pp. 119-136.

[37] Oliveira, F. M., Correa, J. F. S., Lepikson, H. A., Schnitman, L., 2007. Maice- uma ferramenta para modelagem de conhecimento especialista aplicada a automacao de pocos de petroleo. In: CITARE Congresso Ibero-Americano de Inovacao Tecnologica e Areas Estrategicas, Rio de Janeiro.

[38] Olose, E., 2016. Effective maintenance and reliability program in the production of crude oil and natural gas. International Journal of Scientific and Engineering Research 7 (2), 1048-1056.

[39] Panait, L., Luke, S., 2005. Cooperative multi-agent learning: The state of the art. Autonomous agents and multi-agent systems 11 (3), 387-434.

[40] Panja, P., Velasco, R., Pathak, M., Deo, M., 2018. Application of artificial intelligence to forecast hydrocarbon production from shales. Petroleum 4 (1), 75-89.

[41] Paolocci, I., 2018. Artificial intelligence in the automotive industry. Ph.D. thesis, Politecnico di Torino.

[42] Pěchouček, M., Mařík, V., 2008. Industrial deployment of multi-agent technologies: review and selected case studies. Autonomous agents and multi-agent systems 17 (3), 397-431.

[43] Priyadarshy, S., 2017. Iot revolution in oil and gas industry. Internet of Things and Data Analytics Handbook, 513-520.

[44] Qiao, Y., Peng, J., Ge, L., Wang, H., 2017. Application of pso ls-svm forecasting model in oil and gas production forecast. In: Cognitive Informatics \& Cognitive Computing (ICCI* CC), 2017 IEEE 16th International Conference on. IEEE, pp. 470-474.

[45] Ramírez, M. A., Dávila, J., Morles, E. C., La Hechicera, C., 2009. Intelligent supervision based on multi-agent systems: application to petroleum industrial processes. In: Proceedings of the 13th WSEAS international conference on Systems. World Scientific and Engineering Academy and Society (WSEAS), pp. 498-505.

[46] reza Akhondi, M., Talevski, A., Carlsen, S., Petersen, S., 2010. Applications of wireless sensor networks in the oil, gas and resources industries. In: Advanced Information Networking and Applications (AINA), 
2010 24th IEEE International Conference on. IEEE, pp. 941-948.

[47] Sayda, A. F., Taylor, J. H., 2007. An intelligent multi agent system for integrated control and asset management of petroleum production facilities. In: In Proc. of The 17th International Conference on Flexible Automation and Intelligent Manufacturing (FAIM). pp. 851-858.

[48] Sayda, A. F., Taylor, J. H., 2008. A multi-agent system for integrated control and asset management of petroleum production facilities-part 1: Prototype design and development. In: Intelligent Control, 2008. ISIC 2008. IEEE International Symposium on. IEEE, pp. 162-168.

[49] Shukla, A., Karki, H., 2016. Application of robotics in offshore oil and gas industrya review part ii. Robotics and Autonomous Systems 75, 508-524.

[50] Sinha, A. K., Aditya, H., Tiwari, M. K., Chan, F. T., 2009. Multi-agent based petroleum supply chain coordination: A co-evolutionary particle swarm optimization approach. In: Nature and Biologically Inspired Computing, 2009. NaBIC 2009. World Congress on. IEEE, pp. 1349-1354.

[51] Stone, P., Veloso, M., 2000. Multiagent systems: A survey from a machine learning perspective. Autonomous Robots 8 (3), 345-383.

[52] Sutton, R. S., Barto, A. G., 1998. Reinforcement Learning: An Introduction. MIT Press.

[53] Thangarajah, J., Padgham, L., Winikoff, M., 2005. Prometheus design tool. In: 4th International Joint Conference on Autonomous Agents and Multiagent Systems (AAMAS 2005). pp. 127-128.

[54] Tsegha, E., 2013. Assessing the challenges and opportunities in the oil and gas industry. Academic Journal of Interdisciplinary Studies 2 (12), 129.

[55] Velez-Langs, O., 2005. Genetic algorithms in oil industry: An overview. Journal of Petroleum Science and Engineering 47 (1-2), 15-22.

[56] WEC, 2016. World energy resources: Oil 2016. World Energy Council 24, 91.

[57] Weiss, G., 1999. Multiagent systems: a modern approach to distributed artificial intelligence. MIT press.

[58] Wooldridge, M., 2002. An introduction to multi agent systems, department of computer science, university of liverpool, uk. 